\title{
Salidroside inhibits chronic myeloid leukemia cell proliferation and induces apoptosis by regulating the miR-140-5p/wnt5a/ $\beta$-catenin axis
}

\author{
DANJUN CHEN $^{1}$ and CONG LUO ${ }^{2}$ \\ Departments of ${ }^{1}$ Pharmacy and ${ }^{2}$ Hematology, The First Affiliated Hospital of \\ University of South China, Hengyang, Hunan 421001, P.R. China
}

Received April 30, 2020; Accepted February 18, 2021

DOI: $10.3892 /$ etm.2021.10684

\begin{abstract}
Salidroside, an active ingredient of Rhodiola rosea, exhibits antitumor effects in various types of cancer. However, the role of salidroside in chronic myeloid leukemia (CML) has not been elucidated. In the presents study, cell viability was assessed by CCK- 8 assay, while apoptosis was detected by flow cytometry. Reverse transcription-quantitative PCR analysis was used to examine the expression levels of miR-140-5p in human CML cell lines. The expression levels of apoptosis and cell cycle-associated proteins and of the wnt5a/ $\beta$-catenin signaling pathway were determined by western blot analysis. Bioinformatic analysis and luciferase reporter assays were employed to investigate the association between miR-140-5p and wnt5a. The results revealed that exposure of CML cells to salidroside $(80 \mu \mathrm{M})$ inhibited cell proliferation and promoted apoptosis. In addition, salidroside treatment led to the upregulation of miR-140-5p expression. Furthermore, the inhibition of wnt $5 \mathrm{a} / \beta$-catenin signaling pathway and the pro-apoptotic effects induced by salidroside were attenuated by miR-140-5p silencing. Notably, wnt5a was revealed to be a direct target of miR-140-5p. The present findings indicated that salidroside exerted anti-CML effects through regulating miR-140-5p by suppressing the wnt $5 \mathrm{a} / \beta$-catenin signaling pathway. The present study provided evidence of the therapeutic role of salidroside in CML.
\end{abstract}

\section{Introduction}

Chronic myeloid leukemia (CML) is a clonal malignant tumor resulting from hematopoietic disorders, with an incidence of 1-2/10,000 adults per year (1). Currently, the most effective

Correspondence to: Dr Cong Luo, Department of Hematology, The First Affiliated Hospital of University of South China, 69 Chuanshan Road, Hengyang, Hunan 421001, P.R. China

E-mail: luocong1118@126-web.net

Key words: chronic myeloid leukemia, miR-140-5p, salidroside, wnt $5 \mathrm{a} / \beta$-catenin pathway treatment for CML is bone marrow transplantation. However, high risk of mortality and recurrence rates and the difficulty in obtaining a matched donor limit its application (2). Although many patients with CML have a response to hematopoietic stem cell implantation, refractory disease is common, and relapse represents the major cause of treatment failure (3). Therefore, it is urgent to understand the pathogenesis of CML and discover novel effective treatments for CML.

In most cases, CML arises owing to the aberrant formation of a chimeric gene for a constitutively active tyrosine kinase. Thus, tyrosine kinase inhibitors (TKIs) are approved for the first-line treatment of chronic phase CML (4). However, second-generation TKIs have cardiovascular risks that are greater than with imatinib treatment (5). To the best of our knowledge, traditional Chinese medicines or natural products exhibit advantages in tumor prevention and treatment $(6,7)$. Several lines of evidence suggest that salidroside, a major active component of Rhodiola rosea which has been widely used as a tonic herb exhibiting antitumor properties in various cancers, may be a promising novel drug candidate for cancer therapy. For instance, salidroside reduced cell proliferation and induced G1 phase cell cycle arrest and apoptosis of renal cell carcinoma cells (8). In human colorectal cancer HT29 cells, salidroside enhanced autophagic effects by inducing apoptosis via the inactivation of PI3K/Akt/mTOR signaling pathway (9). Salidroside suppressed migration and invasion and induced apoptosis of poorly differentiated thyroid cancer cells via the inhibition of JAK2/STAT3 pathway (10). Furthermore, it has been shown that salidroside inhibited the malignant behaviors of gastric cancer cells (11). Kang et al (12) reported that salidroside induced the angiogenesis, migration and metastasis of breast cancer cells through the STAT3 signaling pathway. Yu et al (13) reported that salidroside was a potent inducer of apoptosis in human ovarian cancer cells through the p53 signaling pathway. Ren et al (14) reported that salidroside had anticarcinogenic activities in human lung cancer cells by suppressing proliferation, migration and invasion through AKT and MEK/ERK signaling. However, the potential antitumor function of salidroside in CML has not been extensively investigated.

The present study aimed to elucidate whether salidroside could be used as a therapeutic agent to modulate the growth 
of CML cell lines and to further evaluate the antitumor potential of salidroside against CML. Considering the anticancer mechanisms of Chinese medicinal herbs targeting microRNAs (miRNAs), we herein examined the potential mechanisms underlying salidroside's antitumor properties by focusing on miRNAs $(15,16)$. miR-140-5p is one of the most studied miRNAs in the field of human cancer $(17,18)$. In regard of CML, miR-140-5p was observed to be downregulated in CML patients and CML cell lines, and the overexpression of miR-140-5p promoted CML cell apoptosis (19). Based on these previous observations, miR-140-5p was selected for further investigation. The findings of the present study may provide evidence for novel therapeutic strategies for CML.

\section{Materials and methods}

Cell lines. CML cell lines, K562 and KCL22, purchased from American Type Culture Collection, were cultured in RPMI-1640 media with fetal bovine serum (FBS), penicillin and streptomycin (all from Gibco; Thermo Fisher Scientific, Inc.) at $37^{\circ} \mathrm{C}$ in a normoxic environment $\left(5 \% \mathrm{CO}_{2}\right.$ and $95 \%$ air). Cells were incubated with $0,20,40,60,80$ and $100 \mu \mathrm{M}$ salidroside (Sigma-Aldrich; Merck KGaA; cat. no. 43866; analytical grade; purity $\geq 98.0 \%$ ) for $12,24,48$ and $72 \mathrm{~h}$. When indicated, cells were transfected with miR-140-5p mimic (5'-GACUAC GAUAUCGAGCCAUA-3'; $50 \mathrm{nM}$ ), inhibitor (5'-UGUACA CACGAUUGACGUG-3'; $100 \mathrm{nM}$ ) or negative controls (NC; miR-NC, 5'-AGUCUAACGCGAGCGUAUAA-3'; $50 \mathrm{nM}$; inhibitor-NC, 5'-GGUAUUACA AAGGUCGCACAA-3'; $100 \mathrm{nM}$ ), which were obtained from Shanghai GenePharma Co., Ltd., by using Lipofectamine 2000 (Thermo Fisher Scientific, Inc.), according to the manufacturer's instructions. After $48 \mathrm{~h}$, the cells were harvested for further experiments.

Cell viability assay. Cell viability was determined by CCK- 8 assay (Beyotime Institute of Biotechnology). Briefly, CCK- 8 solution $(10 \mu \mathrm{l})$ was added to each well at 12, 24, 48 and $72 \mathrm{~h}$. After incubation at $37^{\circ} \mathrm{C}$ for $4 \mathrm{~h}$, the absorbance was detected at $450 \mathrm{~nm}$.

Cell apoptosis assay. K562 and KCL22 cells were inoculated in a six-well plate for $24 \mathrm{~h}$. Next, the culture medium was replaced with a medium containing $10 \%$ FBS. After $24 \mathrm{~h}$, the cells were harvested and stained with Annexin V-fluorescein isothiocyanate (FITC) and propidium iodide (PI) using a double-staining apoptosis detection kit (Invitrogen; Thermo Fisher Scientific, Inc.) following the manufacturer's protocols. Cell apoptosis was determined by flow cytometry analysis using a FACSCanto II flow cytometer (Becton-Dickinson and Company) and calculated using Cell Quest acquisition software (version 2.9; BD Biosciences).

$R N A$ extraction and reverse transcription-quantitative PCR analysis. Total RNA was extracted from K562 and KCL22 cells by using TRIzol reagent (Invitrogen; Thermo Fisher Scientific, Inc.) and was reverse transcribed to cDNA by using MiRcute miRNA first-strand cDNA synthesis kit (Tiangen Biotech Co., Ltd.), according to the manufacturer's protocol. Relative expression levels of miR-140-5p were determined using MiRcute miRNA qPCR detection kit (Tiangen Biotech
Co., Ltd.) on an ABI 7500 real-time PCR system (Applied Biosystems; Thermo Fisher Scientific, Inc.). The thermocycling conditions used for qPCR were as follows: Initial denaturation at $95^{\circ} \mathrm{C}$ for $3 \mathrm{~min}$; followed by 40 cycles of $12 \mathrm{sec}$ at $95^{\circ} \mathrm{C}$ and $40 \mathrm{sec}$ at $62^{\circ} \mathrm{C}$; the fluorescence signal was collected after 40 cycles. Relative fold changes in mRNA expression were calculated using the formula $2^{-\Delta \Delta \mathrm{Cq}}$ method (20). U6 was used as an internal control. The primer sequences were as follows: miR-140-5p, forward, 5'-TGCGGCAGTGGTTTT ACCCTATG-3' and reverse, 5'-CCAGTGCAGGGTCCG AGGT-3'; U6, forward, 5'-CTCGCTTCGGCAGCACATA-3' and reverse, 5'-AACGATTCACGAATTTGCGT-3'.

Western blotting. RIPA lysis buffer (Beyotime Institute of Biotechnology) was used to extract the protein from cultured cells. Total protein concentration was determined using a bicinchoninic acid protein assay kit (Beyotime Institute of Biotechnology) according to the manufacturer's instructions. Equal amounts ( $40 \mu \mathrm{g} / \mathrm{lane})$ of protein were loaded onto a $12 \%$ SDS-PAGE gel and then electroblotted onto polyvinylidene fluoride membranes. Following blocking with 5\% skimmed milk for $2 \mathrm{~h}$ at room temperature, these membranes were incubated with primary antibodies (1:1,000 dilution) overnight at $4^{\circ} \mathrm{C}$. The primary antibodies were (Cell Signaling Technology, Inc.): Cyclin D1 (cat. no. 55506), p21 (cat. no. 2947), Bcl-2 (cat. no. 3498), Bax (cat. no. 5023), pro-caspase-3 (cat. no. 14220), cleaved caspase-3 (cat. no. 9579) and $\beta$-actin (cat. no. 4970). The next day, membranes were incubated at room temperature for $2 \mathrm{~h}$ with FITC-labeled IgG secondary antibodies (cat. no. 7074; 1:2,000 dilution; Cell Signaling Technology, Inc.). The bands were visualized using Novex ECL HRP chemiluminescent substrate reagent kit (Invitrogen; Thermo Fisher Scientific, Inc.) and the protein expression levels were quantified using ImageJ software (version 1.6.0; National Institutes of Health).

Luciferase activity assay. Binding sites for miR-140-5p on the 3'untranslated region (UTR) of wnt5a were identified using the StarBase database (http://starbase.sysu.edu. $\mathrm{cn} /)(21)$ and were confirmed by performing a dual luciferase reporter assay. The 3'-UTR region of wnt5a encompassing the putative miR-140-5p binding site was amplified using PCR and was subsequently sub-cloned into the psiCHECK-2 dual luciferase vector (Promega Corporation). K562 and KCL22 cells were co-transfected with wnt5a recombinant plasmids [wild-type (wnt5a-WT) or mutant (wnt5a-Mut)] and miR-140-5p mimic or miR-NC using Lipofectamine ${ }^{\circledR} 2000$ (Thermo Fisher Scientific, Inc.). After 48 h transfection, luciferase activity was determined using the dual luciferase assay kit after adding firefly or Renilla luciferase reagents (Promega Corporation), according to the manufacturer's instructions.

Statistical analysis. Data were expressed as mean \pm standard deviation. Comparisons were assessed using SPSS 19.0 (IBM Corp.). Differences between two groups were analyzed by Student's t-test. Comparisons between multiple groups were performed by one-way analysis of variance followed by Tukey's post hoc test. $\mathrm{P}<0.05$ was considered to indicate a statistically significant difference. 

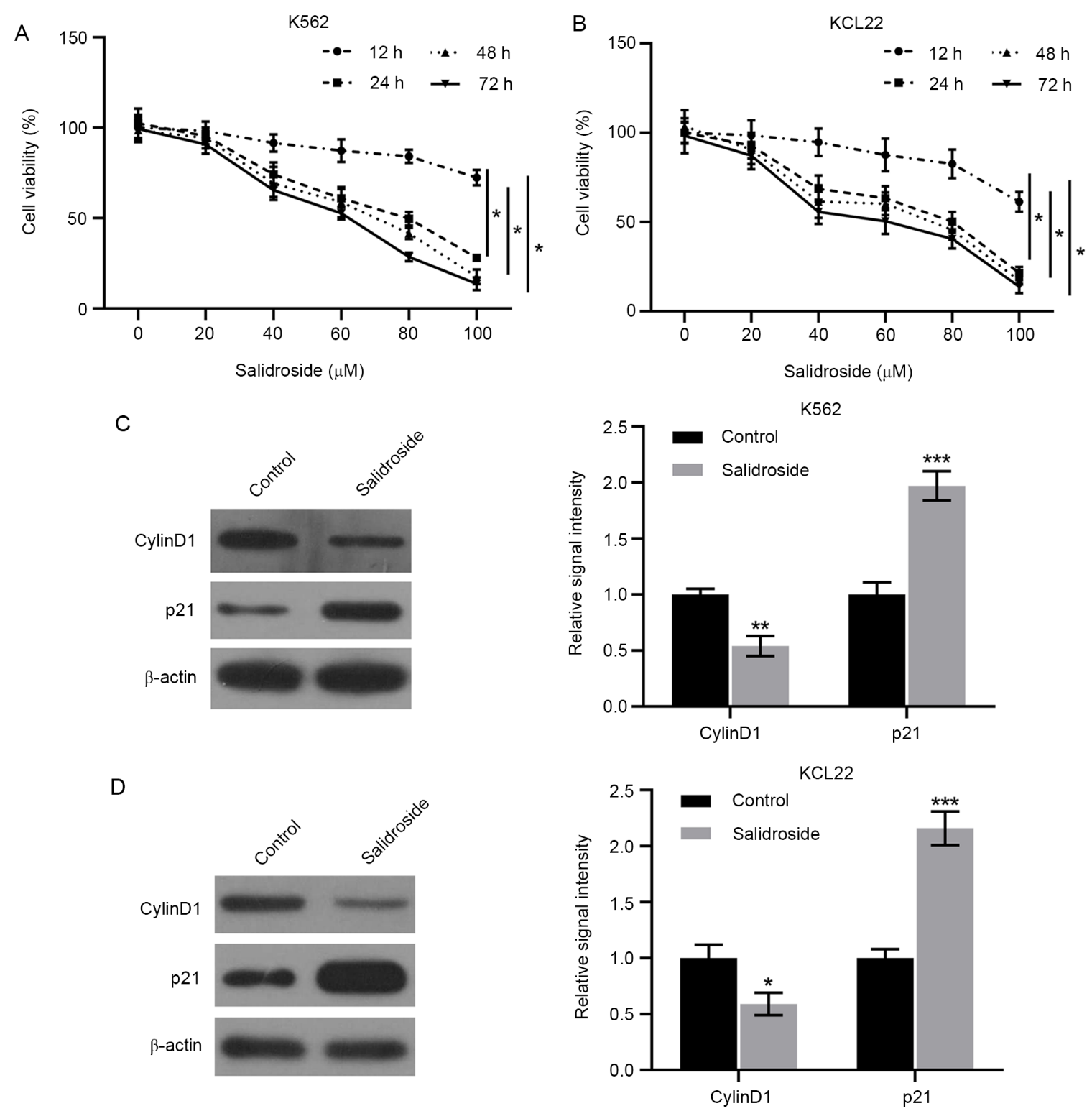

Figure 1. Salidroside inhibits the proliferation and downregulates cell cycle-related protein expression in CML cells. (A) CCK-8 assay was performed to assess cell viability in K562 and (B) KCL22 cells treated with salidroside $(0,20,40,60,80$ and $100 \mu \mathrm{M})$ at different time-points (24, 48 and $72 \mathrm{~h}$ ). (C) The protein expression levels of Cyclin D1 and p21 in K562 and (D) KCL22 cells treated with $80 \mu \mathrm{M}$ salidroside for $24 \mathrm{~h}$ were measured by western blot analysis. "P<0.05, ${ }^{* *} \mathrm{P}<0.01$ and ${ }^{* * *} \mathrm{P}<0.001$ vs. control. CML, chronic myeloid leukemia.

\section{Results}

Salidroside inhibits proliferation and downregulates cell cycle-related protein expression in CML cells. The effects of salidroside on the proliferation and cell cycle-related protein expression of CML cells were first determined. In the present study, human CML cell lines K562 and KCL22 were treated with different concentrations $(0,20,40,60,80$ and $100 \mu \mathrm{M})$ of salidroside for 12, 24, 48 and $72 \mathrm{~h}$. Then, changes in cell viability were monitored by CCK- 8 assay. The results revealed that, compared with the untreated cells, salidroside decreased the numbers of viable K562 (Fig. 1A) and KCL22 (Fig. 1B) cells in a dose and time-dependent manner. Since the $\mathrm{IC}_{50}$ of viability was presented at the concentration of $80 \mu \mathrm{M}$ following $24 \mathrm{~h}$ treatment, the $80 \mu \mathrm{M}$ dose and the $24 \mathrm{~h}$ timepoint were selected for further experimental treatments with salidroside in the present study. Next, changes in expression levels of cell cycle regulatory proteins were assessed. Western blotting revealed that salidroside treatment resulted in the upregulation of p21 and the downregulation of Cyclin D1 expression levels in K562 and KCL22 cells (Fig. 1C and D). These data suggested that salidroside suppressed the proliferation of human CML cells and regulated cell cycle-related proteins.

Salidroside promotes the apoptosis of CML cells. Furthermore, the effects of salidroside on CML cell apoptosis were assessed. The results showed that after treatment with $80 \mu \mathrm{M}$ salidroside for $24 \mathrm{~h}, \mathrm{~K} 562$ and KCL22 cell apoptosis was induced, as demonstrated by flow cytometry analysis with Annexin V-FITC/PI double staining (Fig. 2A and B). In addition, the proapoptotic protein Bax and apoptosis marker protein cleaved caspase-3 were significantly upregulated, while the antiapoptotic protein $\mathrm{Bcl}-2$ expression was decreased, following treatment of K562 and KCL22 cells with salidroside, as evidenced by western 

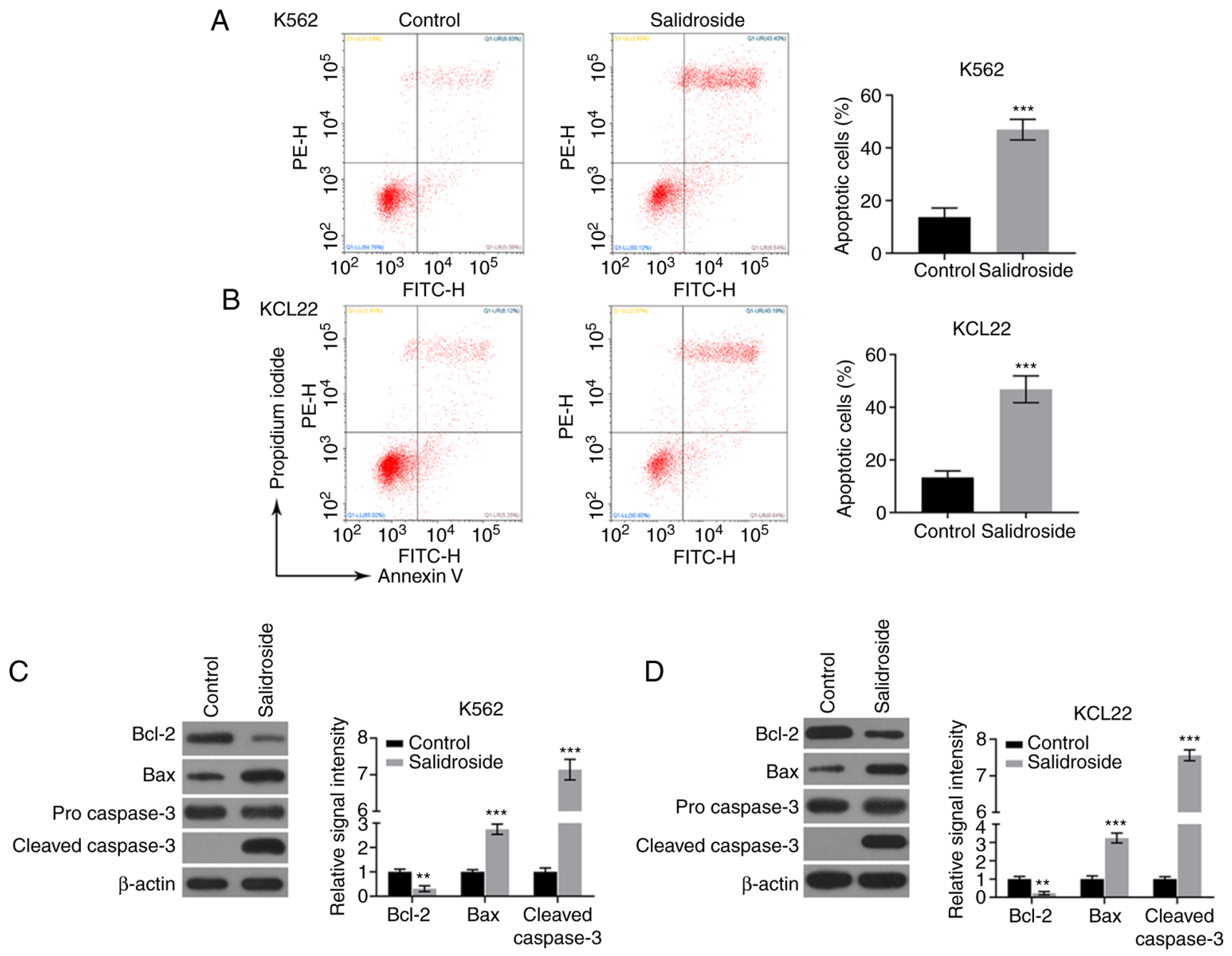

Figure 2. Salidroside promotes the apoptosis of CML cells. K562 and KCL22 cells were treated with $80 \mu \mathrm{M}$ salidroside for 24 h. (A and B) Apoptosis was measured by flow cytometry. (C and D) The protein expression levels of Bcl-2, Bax, pro-caspase-3 and cleaved caspase-3 were determined by western blot; Results for Bcl-2 and Bax were plotted as fold-ratios relative to $\beta$-actin. Results for cleaved caspase-3 were plotted as fold-ratios relative to pro-caspase-3. ${ }^{* *} \mathrm{P}<0.01$ and ${ }^{* * *} \mathrm{P}<0.001$ vs. control. CML, chronic myeloid leukemia.

blotting (Fig. 2C and D). These data indicated that salidroside had proapoptotic effects on cultured human CML cells.

Salidroside affects CML cell proliferation, apoptosis and cell cycle-related protein expression by upregulating miR-140-5p. The results of qPCR analysis demonstrated that, in both CML cell lines, treatment with salidroside resulted in the upregulation of miR-140-5p expression (Fig. 3A). Furthermore, silencing of miR-140-5p expression by using a miR-140-5p-specific inhibitor (Fig. 3B) reversed the effects of salidroside on cell proliferation (Fig. 3C and D), on the Cyclin D1 and p21 protein expression (Fig. 3E and F), on cell apoptosis (Fig. 3G and H), and on the the protein levels of Bcl-2, Bax and cleaved caspase-3 (Fig. 3I and J). Additionally, when miR-140-5p was overexpressed by transfection with a miR-140-5p mimic (Fig. S1A), the cell viability was significantly inhibited (Fig. S1B), while cell apoptosis was significantly increased (Fig. S1C and D) for both K562 and KCL22 cell lines. The present results indicated that salidroside might influence CML cells through the regulation of miR-140-5p.

Salidroside regulates the miR-140-5p/wnt $5 a / \beta$-catenin axis in CML cells. Finally, the potential involvement of miR-140-5p in salidroside-modulated signaling pathways was investigated. The online bioinformatic database StarBase was used to identify potential target genes of miR-140-5p. According to the analysis, wnt5a was predicted to be a potential target gene of miR-140-5p. The predicted binding sites between miR-140-5p and wnt5a are presented in Fig. 4A. In order to investigate whether miR-140-5p regulated wnt5a expression by directly binding to its 3'UTR, a luciferase reporter assay was performed. The results showed that miR-140-5p overexpression significantly decreased the luciferase activity of wnt5a-WT, whereas K562 and KCL22 cells co-transfected with Wnt5a-Mut and miR-140-5p mimic showed no obvious changes in their luciferase activity (Fig. 4B and C). In addition, ectopic expression of miR-140-5p in K562 and KCL22 cells resulted in a decrease of Wnt5a protein expression levels (Fig. 4D). These findings indicated that wnt5a was a direct target of miR-140-5p in CML cells. The present study then explored whether miR-140-5p could mediate the effects of salidroside on the wnt $5 \mathrm{a} / \beta$-catenin pathway. As shown in Fig. 4E and F, the protein expression levels of Wnt5a and $\beta$-catenin were significantly decreased by salidroside. Conversely, miR-140-5p inhibition reversed this effect. 
A

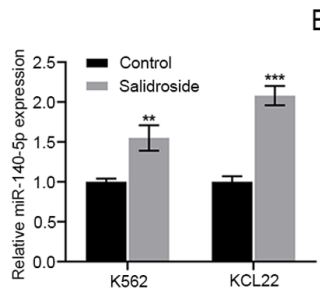

B

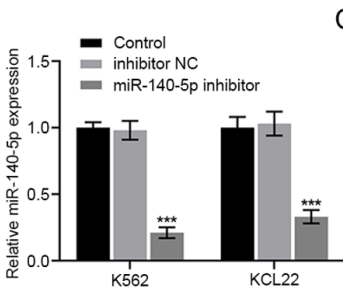

C

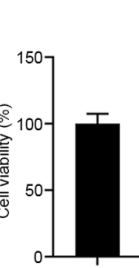

kro
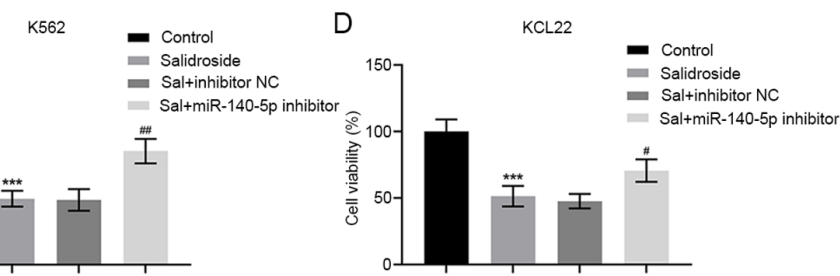

E
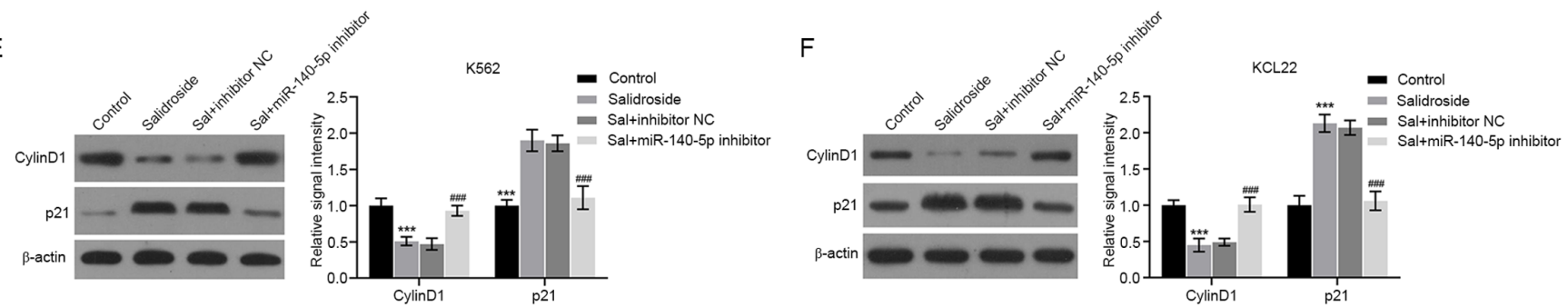

G
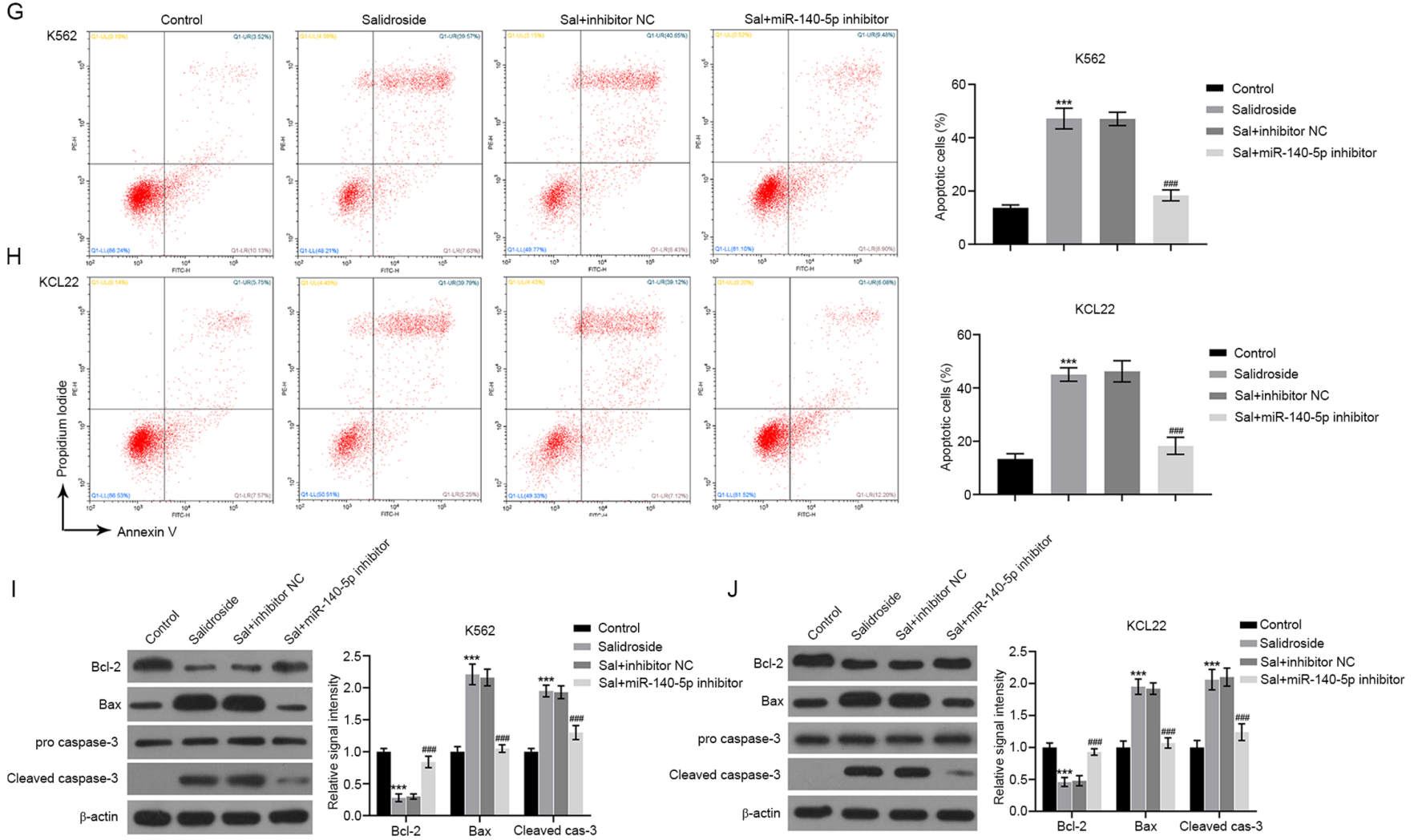

Figure 3. Salidroside affects CML cell proliferation, apoptosis and cell cycle-related protein expression by downregulating miR-140-5p. (A) K562 and KCL22 cells were treated with $80 \mu \mathrm{M}$ salidroside for $24 \mathrm{~h}$. RT-qPCR was then utilized to examine the expression levels of miR-140-5p. ${ }^{* * *} \mathrm{P}<0.01$ and ${ }^{* * * *} \mathrm{P}<0.001$ vs. control. (B) K562 and KCL22 cells were transfected with miR-140-5p inhibitor or inhibitor NC. RT-qPCR was utilized to examine the expression levels of miR-140-5p. ${ }^{* * *} \mathrm{P}<0.001$ vs. inhibitor NC. (C and D) After transfection with miR-140-5p inhibitor or NC for $48 \mathrm{~h}, \mathrm{~K} 562$ and KCL22 cells were stimulated with $80 \mu \mathrm{M}$ salidroside for $24 \mathrm{~h}$. CCK-8 assay was performed to assess cell viability. (E and F) The protein expression levels of Cyclin D1 and p21 were measured by western blotting. (G and H) Apoptosis was measured by flow cytometry. (I and J) The protein expression levels of Bcl-2, Bax, pro-caspase-3 and cleaved caspase-3 were determined by western blotting; Results for Bcl- 2 and Bax were plotted as fold-ratios relative to $\beta$-actin. Results for cleaved caspase- 3 were plotted as fold-ratios relative to pro-caspase-3. ${ }^{* * *} \mathrm{P}<0.001$ vs. control; ${ }^{~} \mathrm{P}<0.05,{ }^{\# \#} \mathrm{P}<0.01$ and ${ }^{\# \# \#} \mathrm{P}<0.001$ vs. Sal + inhibitor NC. CML, chronic myeloid leukemia; RT-qPCR, reverse transcription-quantitative PCR; NC, negative control.

\section{Discussion}

The present study provided the first demonstration of the antitumor mechanism of salidroside in CML. The current findings revealed that salidroside accelerated CML cell apoptosis and inhibited proliferation through downregulation of miR-140-5p and the wnt $5 \mathrm{a} / \beta$-catenin signaling pathway.

A previous study has shown that salidroside inhibited the growth of K562 cells in a dose and time-dependent manner, and induced apoptosis (22). Similarly, the present results demonstrated that salidroside significantly reduced the cell viability of two CML cell lines (K562 and KCL22) in a concentration-dependent manner. In addition, salidroside at $80 \mu \mathrm{M}$ significantly reduced CML cell proliferation, and induced apoptosis. In the cell cycle progression, Cyclin D1, a regulatory subunit of cyclin-dependent kinase (CDK) 4 and CDK6, is required for G1/S transition of the cell cycle to entry into the S-phase (23). p21 is a potent CDK inhibitor by 
A

wnt5a 3'UTR WT: 5' uuuuuuuguguguuuACCACUg 3' hsa-miR-140-5p: $\quad$ ' gaugguaucccauuuUGGUGAc 5' wnt5a 3'UTR MUT: 5' uuuuuuuguguguuuUGAUGAg 3

D
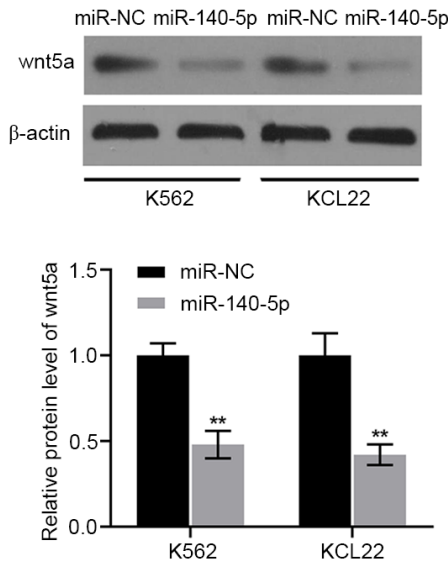

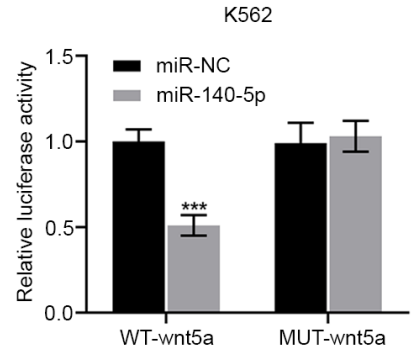

C

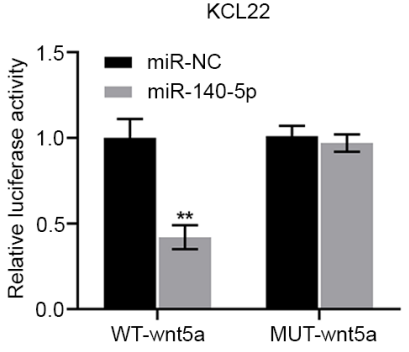

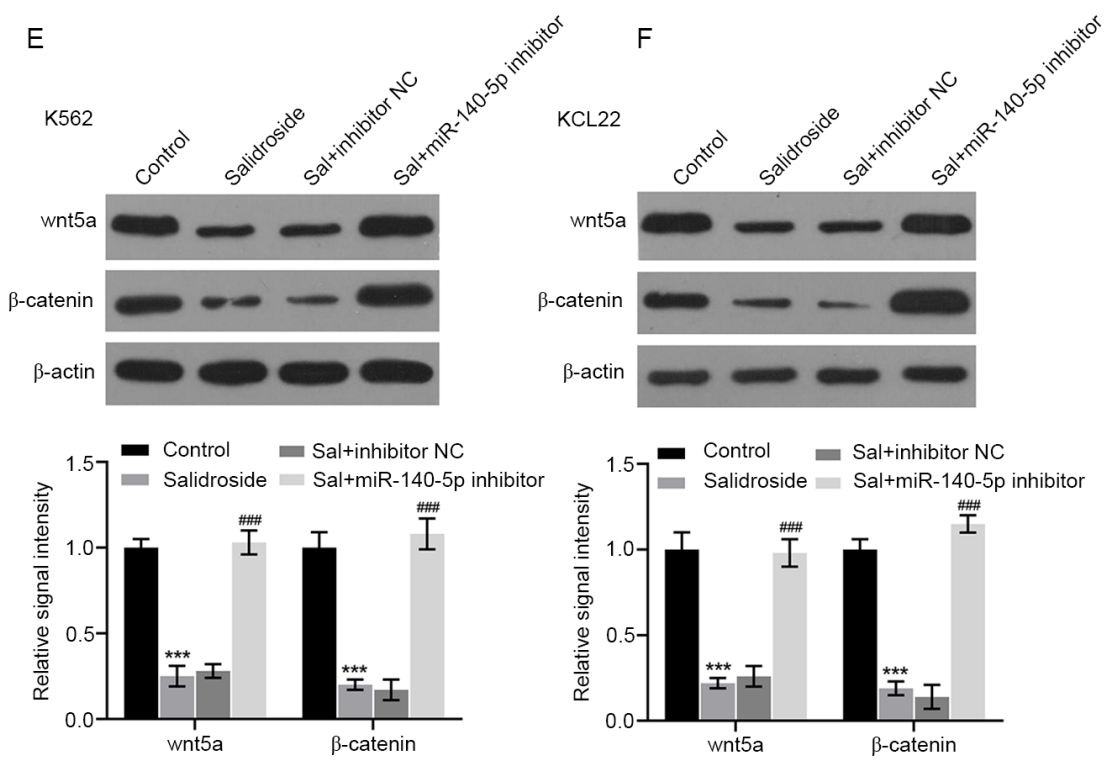

Figure 4. Salidroside regulates the miR-140-5p/wnt5a/ $\beta$-catenin axis in CML cells. (A) The binding sites between miR-140-5p and Wnt5a are illustrated. (B) K562 and (C) KCL22 cells were transfected with wnt5a-WT or MUT reporter plasmid along with miR-140-5p mimic or mimic-NC. Luciferase reporter assay was then performed to investigate the direct binding of miR-140-5p on the wnt5a $3{ }^{\prime} \mathrm{UTR}$. ${ }^{* *} \mathrm{P}<0.01$ and ${ }^{* * * *} \mathrm{P}<0.001$ vs. miR-NC. (D) K562 and KCL22 cells were transfected with miR-140-5p or miR-NC, and the protein expression levels of wnt5a were determined by western blot analysis. ${ }^{* *} \mathrm{P}<0.01 \mathrm{vs}$. miR-NC. (E and F) Following $48 \mathrm{~h}$ transfection with miR-140-5p inhibitor or inhibitor NC, K562 and KCL22 cells were stimulated with $80 \mu \mathrm{M}$ salidroside for $24 \mathrm{~h}$ The protein expression levels of Wnt5a and $\beta$-catenin were determined by western blot analysis. ${ }^{* * * *} \mathrm{P}<0.001$ vs. control; ${ }^{\# \# \#} \mathrm{P}<0.001 \mathrm{vs}$. Sal + inhibitor NC. CML, chronic myeloid leukemia; WT, wild-type, MUT, mutant; NC, negative control; untranslated region.

abrogating all cyclin-CDK complexes and thus functions as a regulator of cell cycle progression at G1 and S phases (24). Qie et al (23) and Ren et al (14) found that salidroside could cause G1-phase arrest, a decrease of cyclin D1 expression and an increase of p21 expression in breast cancer cells and lung cancer cells. The current study revealed that salidroside treatment resulted in a decrease in the expression of Cyclin D1, but an increase in the expression of p21, in K562 and KCL22 cells.

Extensive research on miRNAs has confirmed that they serve important roles in the occurrence and development of CML by acting as oncogenes or tumor suppressors (25). Among them, the antitumor roles of miR-140-5p have been described in multiple types of human cancer by regulating tumor cell proliferation, apoptosis and cell cycle progression (26). A previous study has demonstrated that miR-140-5p was downregulated in CML and its overexpression was associated with the proliferation and apoptosis of CML cells by targeting SIX homeobox 1 (19). Based on this previous literature, the present study speculated that targeting miR-140-5p might be a promising strategy for CML treatment. The present study further revealed that the addition of salidroside upregulated the expression of miR-140-5p in CML cells, implying that miR-140-5p might be implicated in the antitumor effects of salidroside.
Accumulating studies have highlighted the involvement of several signaling pathways in the initiation and development of CML, including NF- $\kappa B$ (27), JAK/STAT (28), and $\mathrm{PI} 3 \mathrm{~K} / \mathrm{AKT} / \mathrm{mTOR}$ (29). In recent years, several reports have proposed that salidroside displays its antitumor activities via the modulation of p53 (13), JAK2/STAT3 (30) and Wnt/ $\beta$-catenin (31) signaling pathways. Cha et al (32) uncovered that miR-140-5p suppressed gastric cancer cell proliferation and invasion by regulating WNT1 expression. Han et al (33) showed that miR-140-5p promoted the cerebral protective effects of dexmedetomidine against hypoxic-ischemic brain damage in neonatal rats by targeting Wnt1 through the negative regulation of the Wnt/ $/$-catenin signaling pathway. Zhao et al (34) reported that miR-140-5p hindered cell proliferation, invasion and tumorigenesis by targeting SRY-box transcription factor 4 via inactivation of the Wnt/ק-catenin and NF- $\kappa \mathrm{B}$ signaling pathways in malignant melanoma. The present study further investigated whether Wnt/ $\beta$-catenin signaling was involved in the antitumor action of salidroside in CML cells, because Wnt5a was identified as a target of miR-140-5p. Additionally, the present results demonstrated that salidroside treatment blocked the activation of the Wnt $5 \mathrm{a} / \beta$-catenin signaling pathway. Notably, the effects of salidroside on the $\mathrm{Wnt} 5 \mathrm{a} / \beta$-catenin signaling pathway were 
partially reversed by miR-140-5p inhibition, suggesting that salidroside blocked the Wnt5a/ $\beta$-catenin signaling pathway possibly through the upregulation of miR-140-5p. The present study has nonetheless several limitations. Firstly, clinical evaluations and in vivo experiments were not performed at this time. Furthermore, a detailed cell cycle analysis is needed to further evaluate the effects of salidroside on K562 and KCL22 cyclin expressions. In addition, other molecules and signaling pathways may be involved in the antitumor effects of salidroside and future studies will be required to elucidate these.

In conclusion, the present findings confirmed the antitumor properties of salidroside in CML via the inhibition of Wnt5a/ $\beta$-catenin signaling pathway by upregulating miR-140-5p expression. Further studies are needed to confirm the potential of salidroside as a therapeutic agent in CML.

\section{Acknowledgements}

Not applicable.

\section{Funding}

This study was funded by a 2019 Guiding project of Hengyang Science and Technology Bureau (no grant number available), a 2019 Hunan Provincial Natural Science Youth Fund (grant no. 2019JJ50536) and a 2018 National Natural Science Youth Fund (grant no. 81803473).

\section{Availability of data and materials}

All data generated or analyzed during this study are included in this published article.

\section{Authors' contributions}

CL designed the study. CL and DC performed the experiments, analyzed the data and prepared the manuscript. CL reviewed the manuscript. All authors read and approved the final manuscript. CL and DC confirm the authenticity of all the raw data. All authors agree to be accountable for all aspects of the research in ensuring that the accuracy or integrity of any part of the work are appropriately investigated and resolved.

\section{Ethics approval and consent to participate}

Not applicable.

\section{Patient consent for publication}

Not applicable.

\section{Competing interests}

The authors declare that they have no competing interests.

\section{References}

1. Flis S and Chojnacki T: Chronic myelogenous leukemia, a still unsolved problem: Pitfalls and new therapeutic possibilities. Drug Des Devel Ther 13: 825-843, 2019.
2. Gupta A and Khattry N: Current status of hematopoietic stem cell transplant in chronic myeloid leukemia. Indian J Med Paediatr Oncol 35: 207-210, 2014.

3. Hino Y, Doki N, Yamamoto K, Senoo Y, Sasajima S, Sakaguchi M, Hattori K, Kaito S, Kurosawa S, Harada K, et al: Chronic myeloid leukemia relapsing ten years after allogenic bone marrow transplantation. Rinsho Ketsueki 57: 608-612, 2016 (In Japanese)

4. Jabbour E and Kantarjian H: Chronic myeloid leukemia: 2016 update on diagnosis, therapy, and monitoring. Am J Hematol 91: 252-265, 2016.

5. Ross DM, Arthur C, Burbury K, Ko BS, Mills AK, Shortt J and Kostner K: Chronic myeloid leukaemia and tyrosine kinase inhibitor therapy: Assessment and management of cardiovascular risk factors. Intern Med J 48 (Suppl 2): S5-S13, 2018.

6. Li H, Liu L, Liu C, Zhuang J, Zhou C, Yang J, Gao C, Liu G, Lv Q and Sun C: Deciphering key pharmacological pathways of qingdai acting on chronic myeloid leukemia using a network pharmacology-based strategy. Med Sci Monit 24: 5668-5688, 2018.

7. Zhang Y, Xiao Y, Dong Q, Ouyang W and Qin Q: Neferine in the lotus plumule potentiates the antitumor effect of imatinib in primary chronic myeloid leukemia cells in vitro. J Food Sci 84 904-910, 2019

8. Lv C, Huang Y, Liu ZX, Yu D and Bai ZM: Salidroside reduces renal cell carcinoma proliferation by inhibiting JAK2/STAT3 signaling. Cancer Biomark 17: 41-47, 2016.

9. Fan XJ, Wang Y, Wang L and Zhu M: Salidroside induces apoptosis and autophagy in human colorectal cancer cells through inhibition of PI3K/Akt/mTOR pathway. Oncol Rep 36: 3559-3567, 2016.

10. Shang H, Wang S, Yao J, Guo C, Dong J and Liao L: Salidroside inhibits migration and invasion of poorly differentiated thyroid cancer cells. Thorac Cancer 10: 1469-1478, 2019.

11. Qi Z, Tang T, Sheng L, Ma Y, Liu Y, Yan L, Qi S, Ling L and Zhang Y: Salidroside inhibits the proliferation and migration of gastric cancer cells via suppression of Src-associated signaling pathway activation and heat shock protein 70 expression. Mol Med Rep 18: 147-156, 2018.

12. Kang DY, Sp N, Kim DH, Joung YH, Lee HG, Park YM and Yang YM: Salidroside inhibits migration, invasion and angiogenesis of MDAMB 231 TNBC cells by regulating EGFR/Jak2/STAT3 signaling via MMP2. Int J Oncol 53: 877-885, 2018.

13. Yu G, Li N, Zhao Y, Wang W and Feng XL: Salidroside induces apoptosis in human ovarian cancer SKOV3 and A2780 cells through the p53 signaling pathway. Oncol Lett 15: 6513-6518, 2018.

14. Ren $\mathrm{M}, \mathrm{Xu} \mathrm{W}$ and $\mathrm{Xu} \mathrm{T}$ : Salidroside represses proliferation, migration and invasion of human lung cancer cells through AKT and MEK/ERK signal pathway. Artif Cells Nanomed Biotechnol 47: 1014-1021, 2019.

15. Yang L, Yu Y, Zhang Q, Li X, Zhang C, Mao T, Liu S and Tian Z: Anti-gastric cancer effect of Salidroside through elevating miR-99a expression. Artif Cells Nanomed Biotechnol 47: 3500-3510, 2019.

16. Li H, Huang D and Hang S: Salidroside inhibits the growth, migration and invasion of Wilms' tumor cells through down-regulation of miR-891b. Life Sci 222: 60-68, 2019.

17. Wu Y, Li J, Chen S and Yu Z: The effects of miR-140-5p on the biological characteristics of ovarian cancer cells through the Wnt signaling pathway. Adv Clin Exp Med 29: 777-784, 2020.

18. Fang Z, Yin S, Sun R, Zhang S, Fu M, Wu Y, Zhang T, Khaliq J and Li Y: miR-140-5p suppresses the proliferation, migration and invasion of gastric cancer by regulating YES1. Mol Cancer 16: 139,2017

19. Nie ZY, Liu XJ, Zhan Y, Liu MH, Zhang XY, Li ZY, Lu YQ, Luo JM and Yang L: miR-140-5p induces cell apoptosis and decreases Warburg effect in chronic myeloid leukemia by targeting SIX1. Biosci Rep 39: BSR20190150, 2019.

20. Livak KJ and Schmittgen TD: Analysis of relative gene expression data using real-time quantitative PCR and the 2(-Delta Delta $\mathrm{C}(\mathrm{T})$ ) method. Methods 25: 402-408, 2001

21. Li JH, Liu S, Zhou H, Qu LH and Yang JH: StarBase v2.0: Decoding miRNA-ceRNA, miRNA-ncRNA and protein-RNA interaction networks from large-scale CLIP-Seq data. Nucleic Acids Res 42: D92-D97, 2014.

22. Jin YL, Zhang XM, Chen X, Liu J, Chang YY, Gao XY, Xue YM, Dong XS, Liu Y, Tian YY, et al: Salidroside induces apoptosis via ERK1/2 in human leukemia K562 cells. 9: $17588-17595,2016$. 
23. Qie S and Diehl JA: Cyclin D1, cancer progression, and opportunities in cancer treatment. J Mol Med (Berl) 94: 1313-1326, 2016.

24. El-Deiry WS: p21(WAF1) mediates cell-cycle inhibition, relevant to cancer suppression and therapy. Cancer Res 76: 5189-5191, 2016

25. Litwinska $Z$ and Machalinski B: miRNAs in chronic myeloid leukemia: Small molecules, essential function. Leuk Lymphoma 58: 1297-1305, 2017.

26. Liao Y, Yin X, Deng Y and Peng X: MiR-140-5p suppresses retinoblastoma cell growth via inhibiting c-Met/AKT/mTOR pathway. Biosci Rep: Nov 30, 2018 (Epub ahead of print). doi: 10.1042/BSR20180776.

27. Jia Q, Sun H, Xiao F, Sai Y, Li Q, Zhang X, Yang S, Wang H, Wang H, Yang Y, et al: miR-17-92 promotes leukemogenesis in chronic myeloid leukemia via targeting A20 and activation of NF- $\kappa \mathrm{B}$ signaling. Biochem Biophys Res Commun 487: 868-874, 2017.

28. Cai H, Qin X and Yang C: Dehydrocostus lactone suppresses proliferation of human chronic myeloid leukemia cells through Bcr/Abl-JAK/STAT signaling pathways. J Cell Biochem 118: 3381-3390, 2017.

29. Li L, Qi Y, Ma X, Xiong G, Wang L and Bao C: TRIM22 knockdown suppresses chronic myeloid leukemia via inhibiting PI3K/Akt/mTOR signaling pathway. Cell Biol Int 42: $1192-1199,2018$

30. Huang L, Huang Z, Lin W, Wang L, Zhu X, Chen X, Yang S and Lv C: Salidroside suppresses the growth and invasion of human osteosarcoma cell lines MG63 and U2OS in vitro by inhibiting the JAK2/STAT3 signaling pathway. Int J Oncol 54: 1969-1980, 2019.
31. Zhao J, Du X, Wang M, Yang P and Zhang J: Salidroside mitigates hydrogen peroxide-induced injury by enhancement of microRNA-27a in human trabecular meshwork cells. Artif Cells Nanomed Biotechnol 47: 1758-1765, 2019.

32. Cha Y, He Y, Ouyang K, Xiong H, Li J and Yuan X: MicroRNA-140-5p suppresses cell proliferation and invasion in gastric cancer by targeting WNT1 in the $\mathrm{WNT} / \beta$-catenin signaling pathway. Oncol Lett 16: 6369-6376, 2018.

33. Han XR, Wen X, Wang YJ, Wang S, Shen M, Zhang ZF, Fan SH, Shan Q, Wang L, Li MQ, et al: MicroRNA-140-5p elevates cerebral protection of dexmedetomidine against hypoxic-ischaemic brain damage via the $\mathrm{Wnt} / \beta$-catenin signalling pathway. J Cell Mol Med 22: 3167-3182, 2018.

34. Zhao G, Yin Y and Zhao B: miR-140-5p is negatively correlated with proliferation, invasion, and tumorigenesis in malignant melanoma by targeting SOX4 via the Wnt/ $\beta$-catenin and NF- $\kappa \mathrm{B}$ cascades. J Cell Physiol 235: 2161-2170, 2020.

This work is licensed under a Creative Commons Attribution-NonCommercial-NoDerivatives 4.0 International (CC BY-NC-ND 4.0) License. 